\title{
New Diels-Alder Type Adducts from Morus macroura and Their Anti-oxidant Activities
}

\author{
Sheng-Jun DAI, Yan Wu, Ying-Hong WANG, Wen-Yi He, Ruo-Yun Chen, and De-Quan Yu* \\ Institute of Materia Medica, Chinese Academy of Medical Sciences \& Peking Union Medical College; Beijing 100050, \\ P. R. China. Received May 17, 2004; accepted July 5, 2004
}

\begin{abstract}
Fractionation of the ethanolic extract of the stem bark of Morus macroura resulted in the isolation of four new Diels-Alder type adducts, named guangsangons $\mathrm{K}-\mathrm{N}(1,2,5,6)$, together with two known compounds, mulberrofuran $G$ (3) and $K(4)$. Their structures were determined on the basis of spectroscopic analyses and chemical methods. Furthermore, by means of ${ }^{1} \mathrm{H}-\mathrm{NMR}$ variable temperature experiments and the Cotton curves in the circular dichroism (CD) spectra, the stereochemistry of four new compounds was elucidated. The isolated new compounds showed good activity on anti-oxidant in vitro, with the inhibitory rates of MDA being from 91.8 to $100.0 \%$ at concentrations of $10^{-5} \mathrm{~mol} / \mathrm{l}$.
\end{abstract}

Key words Moraceae; Morus macroura; guangsangon K; guangsangon L; guangsangon M; guangsangon N

Morus macroura MiQ. belongs to the economically and medically important genus Morus, because its leaves have been an indispensable food source for silk-worms, and its root barks have been used to treat diabetes, arthritis, and rheumatism in Chinese herbal medicine. ${ }^{1)}$ Previously, many novel compounds, which were regarded biogenetically as Diels-Alder adducts of dehydroprenylphenols and chalcone derivatives, were isolated from M. alba, M. lhou, M. bomby$c i s$, and so on. ${ }^{2-8)}$ In the course of our seeking novel bioactive compound, we started phytochemical research on the stem bark of M. macroura. The EtOH extract of M. macroura was partitioned with $\mathrm{CHCl}_{3}$ and EtOAc, and the EtOAc fraction was sequentially subjected to column chromatography over silica gel, silica gel RP-18 and Sephadex LH-20 to give six compounds. The present paper deals with the isolation and structure elucidation of these compounds, as well as the evaluation of anti-oxidant effect of new compounds.

\section{Results and Discussion}

Guangsangon K (1) was obtained as a brown amorphous powder, and gave a dark green color with the methanolic ferric chloride test, and reacted positively to the magnesium hydrochloric acid test. The molecular formula was determined to be $\mathrm{C}_{35} \mathrm{H}_{30} \mathrm{O}_{11}$ by HR-FAB-MS, which showed a quasi-molecular ion peak at $m / z 627.1873[\mathrm{M}+\mathrm{H}]^{+}$. The UV spectrum exhibited maxima at 232 (sh), 278 and 319 (sh) nm, and showed a bathochromic shift in the presence of aluminum chloride, indicating the presence of a chelated carbonyl group. ${ }^{9)}$ The IR spectrum displayed absorption bands at $3402,1674,1612,1502$ and $1462 \mathrm{~cm}^{-1}$ assignable to hydroxyl, conjugated carbonyl and benzene ring moieties. The ${ }^{1} \mathrm{H}-\mathrm{NMR}$ spectrum of $\mathbf{1}$ displayed the following proton sig- nals, a singlet at $\delta 13.32\left(1 \mathrm{H}, \mathrm{s}, \mathrm{HO}-10^{\prime \prime}\right)$ for a chelated hydroxyl group; one set of $\mathrm{ABX}$ aromatic protons assignable to a 2,4-dihydroxyphenyl moiety at $\delta 6.23(\mathrm{~d}, J=2.5 \mathrm{~Hz}, \mathrm{H}-$ $\left.17^{\prime \prime}\right), 6.11$ (dd, $\left.J=2.5,9.0 \mathrm{~Hz}, \mathrm{H}-19^{\prime \prime}\right)$ and $6.88(\mathrm{~d}, J=9.0 \mathrm{~Hz}$, $\left.\mathrm{H}-20^{\prime \prime}\right)$; another set of $\mathrm{ABX}$ aromatic protons assignable to the 2,4-dihydroxybenzoyl unit at $\delta 5.96(\mathrm{~d}, J=2.0 \mathrm{~Hz}, \mathrm{H}-$ $\left.11^{\prime \prime}\right), 6.22\left(\mathrm{dd}, J=2.0,9.0 \mathrm{~Hz}, \mathrm{H}-13^{\prime \prime}\right)$ and $7.46(\mathrm{~d}, J=9.0 \mathrm{~Hz}$, $\left.\mathrm{H}-14^{\prime \prime}\right)$; other set of $\mathrm{ABX}$ aromatic protons at $\delta 6.72$ (d, $J=2.5 \mathrm{~Hz}, \mathrm{H}-8), 6.59$ (dd, $J=2.5,9.0 \mathrm{~Hz}, \mathrm{H}-6)$ and 7.74 (d, $J=9.0 \mathrm{~Hz}, \mathrm{H}-5)$, a pair of ortho-coupled aromatic protons at $\delta 6.25\left(\mathrm{~d}, J=8.5 \mathrm{~Hz}, \mathrm{H}-5^{\prime}\right)$ and $7.09\left(\mathrm{~d}, J=8.5 \mathrm{~Hz}, \mathrm{H}-6^{\prime}\right)$, as well as a pair of vicinal-coupled aliphatic protons at $\delta 4.72$ (d, $J=12.0 \mathrm{~Hz}, \mathrm{H}-3)$ and 5.48 (d, $J=12.0 \mathrm{~Hz}, \mathrm{H}-2)$ attributable to the dihydroflavonol moiety; and protons for a trisubstituted methylcyclohexene ring at $\delta 5.54\left(\mathrm{~s}, \mathrm{H}-2^{\prime \prime}\right), 4.53$ (br s, H-3"), 4.55 (br s, H-4"), 3.72 (br s, H-5"), 2.67 (m, H$\left.6^{\prime \prime}\right), 2.22\left(\mathrm{~m}, \mathrm{H}-6^{\prime \prime}\right)$ and $1.82\left(3 \mathrm{H}, \mathrm{s}, \mathrm{H}-7^{\prime \prime}\right)$. The long-range correlation between $\mathrm{H}-20^{\prime \prime}$ and $\mathrm{C}-5^{\prime \prime}$ suggested that the 2,4-dihydroxyphenyl moiety was connected to the methylcyclohexene ring at C-5". Therefore, the 2,4-dihydroxybenzoyl and dihydroflavonol moieties were connected to the methylcyclohexene ring at C-4" and C-3".

In the ${ }^{1} \mathrm{H}-\mathrm{NMR}$ spectrum $\left(\mathrm{CD}_{3} \mathrm{COCD}_{3}\right)$, the proton signals of the methylcyclohexene ring were broadened, this phenomenon suggested that $\mathbf{1}$ existed as an equilibrium mixture of conformational isomers in solution, ${ }^{3)}$ and variable temperature studies were then carried out. Measured in DMSO- $d_{6}$ at $100{ }^{\circ} \mathrm{C}$, the ${ }^{1} \mathrm{H}-\mathrm{NMR}$ spectrum of the methylcyclohexene ring was resolvable as shown in Fig. 1. Both coupling constants of $\mathrm{H}-4^{\prime \prime} / \mathrm{H}-5^{\prime \prime}$ and $\mathrm{H}-4^{\prime \prime} / \mathrm{H}-3^{\prime \prime}$ were observed to be $10.0 \mathrm{~Hz}$, this suggested H-3", H-4" and H-5" to be axial and the relative configurations of three protons were all-trans.

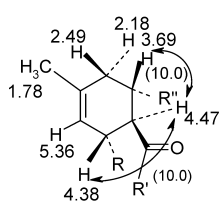

1

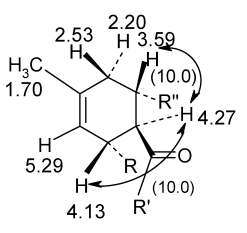

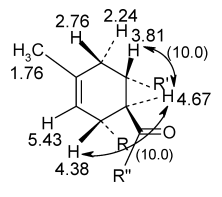

5

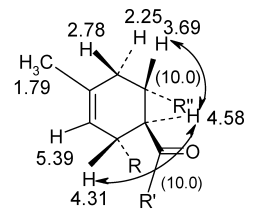

Fig. 1. The Chemical Shifts and Key Coupling Constants (in Parentheses) of the Methylcyclohexene Ring for $\mathbf{1}, \mathbf{2}, \mathbf{5}$ and $\mathbf{6}$ (in DMSO- $d_{6}$, at $100^{\circ} \mathrm{C}$ ) 


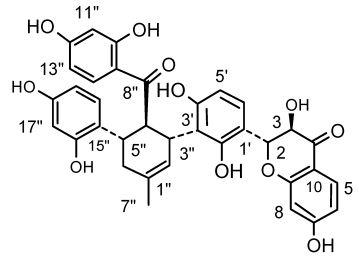

1

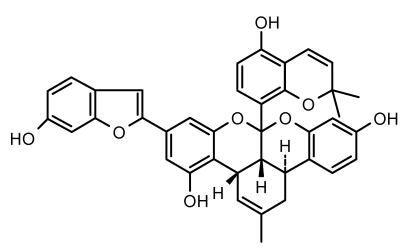

4

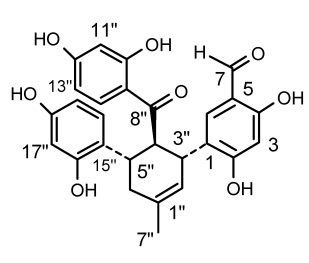

2

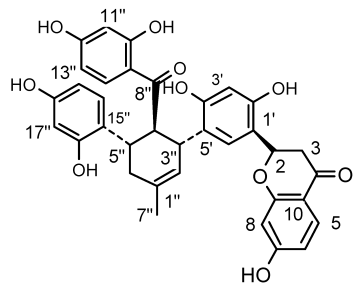

5

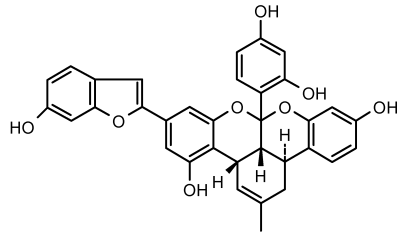

3

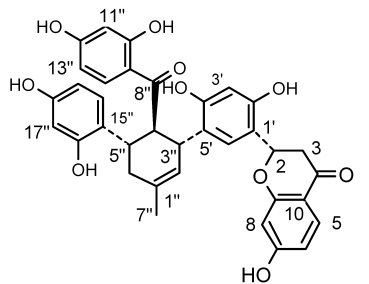

6

Chart 1. The Chemical Structures of 1, 2, 3, 4, 5 and $\mathbf{6}$ from Morus macroura

The stereochemistry of $\mathbf{1}$ with the absolute configurations of $R(\mathrm{C}-2), R(\mathrm{C}-3), R\left(\mathrm{C}-3^{\prime \prime}\right), R\left(\mathrm{C}-4^{\prime \prime}\right)$ and $S\left(\mathrm{C}-5^{\prime \prime}\right)$ was determined by $\mathrm{CD}$ spectrum, which showed a positive Cotton effect at $347 \mathrm{~nm}$, along with two negative Cotton effects at 282 and $287 \mathrm{~nm} \cdot{ }^{10,11)}$

Guangsangon L (2) was isolated and purified as a yellow amorphous powder. The HR-FAB-MS showed a quasi-molecular ion peak at $m / z 477.1558[\mathrm{M}+\mathrm{H}]^{+}$, consistent with a molecular formula of $\mathrm{C}_{27} \mathrm{H}_{24} \mathrm{O}_{8}$. A comparison of NMR $\left({ }^{1} \mathrm{H},{ }^{13} \mathrm{C}\right)$ spectra of $\mathbf{2}$ with those of $\mathbf{1}$ indicated the structure of $\mathbf{2}$ differed from $\mathbf{1}$ only in the moiety at C-3"of the methylcyclohexene ring. Instead of a dihydroflavonol moiety of $\mathbf{1}$, a 2,4-dihydroxybenzaldehyde moiety was present in $\mathbf{2}$. In the ${ }^{1} \mathrm{H}-{ }^{1} \mathrm{H}$ COSY spectrum (measured in DMSO- $d_{6}, 100^{\circ} \mathrm{C}$ ), $\mathrm{H}-4^{\prime \prime}$ was observed to be coupled with $\mathrm{H}-3^{\prime \prime}(J, 10.0 \mathrm{~Hz})$ and also with $\mathrm{H}-5^{\prime \prime}(J, 10.0 \mathrm{~Hz})$, this suggested that three protons were all axial and their relative configurations were all-trans. In the previous paper, ${ }^{10)}$ it was reported that the absolute configurations of three chiral centers on the methylcyclohexene ring in the all-trans adducts were established as $R\left(\mathrm{C}-3^{\prime \prime}\right), R$ $\left(\mathrm{C}-4^{\prime \prime}\right)$ and $S\left(\mathrm{C}-5^{\prime \prime}\right)$, while in the cis-trans adducts as $S$ (C$\left.3^{\prime \prime}\right), R\left(\mathrm{C}-4^{\prime \prime}\right)$ and $S\left(\mathrm{C}-5^{\prime \prime}\right)$. Furthermore, the all-trans adducts exhibited negative optical rotation values while the cis-trans adducts showed positive ones. Since $\mathbf{2}$ displayed negative optical rotation value, the absolute configurations of three unsymmetrical carbons were assigned as $R\left(\mathrm{C}-3^{\prime \prime}\right), R\left(\mathrm{C}-4^{\prime \prime}\right)$ and $S\left(\mathrm{C}-5^{\prime \prime}\right)$, respectively.

Guangsangon M (5) was obtained as a brown amorphous powder, and gave a dark green color with the methanolic ferric chloride test, and was positive to the magnesium hydrochloric acid test. The molecular formula was assigned as $\mathrm{C}_{35} \mathrm{H}_{30} \mathrm{O}_{10}$ by HR-FAB-MS, which showed a quasi-molecular ion peak at $m / z 611.1962[\mathrm{M}+\mathrm{H}]^{+}$. By means of NMR $\left({ }^{1} \mathrm{H}\right.$, ${ }^{13} \mathrm{C}$ ) data, the structure of $\mathbf{5}$ was found to be identical with $\mathbf{1}$ except for the difference of moiety at C-3" of the methylcyclohexene ring. In contrast to the dihydroflavonol moiety of 1, a flavanone unit was connected to the methylcyclohexene ring of 5. ${ }^{1} \mathrm{H}-\mathrm{NMR}$ variable temperature experiment (Fig. 1) showed the relative configurations of $\mathrm{H}-3^{\prime \prime}, \mathrm{H}-4^{\prime \prime}$ and $\mathrm{H}-5^{\prime \prime}$ were all-trans. In the CD spectrum, $\mathbf{5}$ gave a positive Cotton
Table 1. ${ }^{13} \mathrm{C}-\mathrm{NMR}$ Data of $\mathbf{1}, \mathbf{2}, 3$ and $\mathbf{6}$ in Acetone- $d_{6}\left(125 \mathrm{MHz}, 25^{\circ} \mathrm{C}, \delta\right.$ in ppm)

\begin{tabular}{rrrrr}
\hline \hline No. & $\mathbf{1}$ & $\mathbf{2}$ & $\mathbf{5}$ & $\mathbf{6}$ \\
\hline 1 & & 124.9 & & \\
2 & 79.3 & 163.4 & 75.3 & 75.5 \\
3 & 72.8 & 104.0 & 43.3 & 43.4 \\
4 & 193.7 & 164.9 & 190.3 & 190.2 \\
5 & 128.4 & 116.0 & 129.6 & 128.5 \\
6 & 112.0 & 136.2 & 110.7 & 110.5 \\
7 & 165.6 & 195.6 & 165.1 & 165.0 \\
8 & 104.1 & & 103.2 & 103.2 \\
9 & 165.1 & & 164.4 & 164.7 \\
10 & 117.1 & & 114.4 & 114.7 \\
$1^{\prime}$ & 117.4 & & 117.6 & 117.6 \\
$2^{\prime}$ & 156.1 & & 155.8 & 155.7 \\
$3^{\prime}$ & 109.4 & & 102.1 & 102.6 \\
$4^{\prime}$ & 157.4 & & 153.4 & 153.2 \\
$5^{\prime}$ & 108.5 & & 121.9 & 122.0 \\
$6^{\prime}$ & 128.2 & & 128.2 & 128.0 \\
$1^{\prime \prime}$ & 134.1 & 135.1 & 133.6 & 133.7 \\
$2^{\prime \prime}$ & 125.2 & 125.3 & 125.6 & 125.5 \\
$3^{\prime \prime}$ & 47.0 & 42.2 & 41.4 & 43.4 \\
$4^{\prime \prime}$ & 49.7 & 50.9 & 50.1 & 50.8 \\
$5^{\prime \prime}$ & 47.0 & 42.2 & 41.4 & 43.4 \\
$6^{\prime \prime}$ & 37.7 & 37.7 & 37.6 & 37.2 \\
$7^{\prime \prime}$ & 23.5 & 23.5 & 22.7 & 22.7 \\
$8^{\prime \prime}$ & 208.7 & 209.0 & 208.7 & 208.6 \\
$9^{\prime \prime}$ & 115.8 & 115.8 & 115.0 & 115.1 \\
$10^{\prime \prime}$ & 166.6 & 166.2 & 165.1 & 165.1 \\
$11^{\prime \prime}$ & 102.7 & 102.9 & 102.8 & 102.1 \\
$12^{\prime \prime}$ & 165.8 & 163.9 & 164.7 & 164.4 \\
$13^{\prime \prime}$ & 108.2 & 107.8 & 106.8 & 106.9 \\
$14^{\prime \prime}$ & 129.8 & 134.0 & 128.7 & 128.7 \\
$15^{\prime \prime}$ & 121.8 & 121.6 & 121.3 & 121.2 \\
$16^{\prime \prime}$ & 156.6 & 157.5 & 156.6 & 156.5 \\
$17^{\prime \prime}$ & 104.0 & 103.0 & 103.3 & 103.2 \\
$18^{\prime \prime}$ & 156.5 & 156.7 & 155.5 & 155.3 \\
$19^{\prime \prime}$ & 107.6 & 107.6 & 107.4 & 107.2 \\
$20^{\prime \prime}$ & 134.2 & 133.1 & 133.8 & 133.8 \\
& & & & \\
& & & & \\
\hline
\end{tabular}

effect at $305 \mathrm{~nm}$, along with two negative Cotton effects at 285 and $326 \mathrm{~nm}$, and corresponding to the absolute configurations of $R(\mathrm{C}-2), R\left(\mathrm{C}-3^{\prime \prime}\right), R\left(\mathrm{C}-4^{\prime \prime}\right)$ and $S\left(\mathrm{C}-5^{\prime \prime}\right) .^{10,11,13)}$

Guangsangon N (6) was obtained as a brown amorphous 
powder. The molecular formula was determined to be $\mathrm{C}_{35} \mathrm{H}_{30} \mathrm{O}_{10}$ by HR-FAB-MS, which displayed a quasi-molecular ion peak at $m / z 611.1958[\mathrm{M}+\mathrm{H}]^{+}$. Similar to compound 5, the NMR $\left({ }^{1} \mathrm{H},{ }^{13} \mathrm{C}\right)$ spectra of $\mathbf{6}$ also exhibited signals assignable to the 2,4-dihydroxyphenyl, 2,4-dihydroxybenzoyl and flavanone moieties. According to the results of ${ }^{1} \mathrm{H}-\mathrm{NMR}$ variable temperature experiment (Fig. 1) and $\mathrm{CD}$ spectrum (with three negative Cotton effects at 269, 288 and $303 \mathrm{~nm}$, and a positive Cotton effect at $333 \mathrm{~nm}$ ), the relative configurations of $\mathrm{H}-3^{\prime \prime}, \mathrm{H}-4^{\prime \prime}$ and $\mathrm{H}-5^{\prime \prime}$ were assigned as all-trans, and the absolute configurations of C-3", C-4", C-5" and C-2 were assigned as $R, R, S$ and $S$, respectively. ${ }^{10-12)}$

In the case of the bioactivities of compounds $\mathbf{1 , 2 , 5 , 6}$ and Vit $\mathrm{E}$, the inhibitory rates of MDA were $91.8 \%, 97.6 \%$, $98.3 \%, 100.0 \%$ and $18.2 \%$ at concentrations of $10^{-5} \mathrm{~mol} / 1$, respectively. Thus, it could be concluded that these compounds had good anti-oxidant activities.

Phytochemical study of this plant also led to the isolation of two known constituents, mulberrofuran $\mathrm{G}^{14)}(3)$ and $\mathrm{K}^{15)}$ (4), and they were identified by comparison of their physical and spectral data $\left([\alpha]_{\mathrm{D}}, \mathrm{UV}, \mathrm{IR},{ }^{1} \mathrm{H}-\mathrm{NMR},{ }^{13} \mathrm{C}-\mathrm{NMR}, \mathrm{MS}\right)$ with reported values or those of commercial samples.

\section{Experimental}

General Procedures UV spectra were obtained on a Shimadzu UV-160 spectrophotometer. NMR spectra were recorded on a Varian Unity INOVA 500 at $500 \mathrm{MHz}\left({ }^{1} \mathrm{H}\right)$ and $125 \mathrm{MHz}\left({ }^{13} \mathrm{C}\right)$, with TMS as the internal standard. IR spectra were recorded on a Perkin-Elmer 683 infrared spectrometer with $\mathrm{KBr}$ disks. Optical rotations were measured on a Perkin-Elmer 241 polarimeter. FAB-MS and HR-FAB-MS were recorded on an Autospec-Ultima ETOF MS spectrometer. CD spectra were on a JASCO J-725 spectrometer. Silica gel (200-300 mesh) for column chromatography and silica gel GF254 for preparative TLC were obtained from Qingdao Marine Chemical Factory, Qingdao, People's Republic of China. Precoated plates of silica gel GF254 and silica gel RP-18 F254s were used for TLC, and detected under UV. HPLC separation was performed on CONSTA METRIC 3200 and a UV detector at $254 \mathrm{~nm}$.

Plant Material Morus macroura MiQ. was collected in Xishuangbanna, Yunnan Province, Peoples Republic of China, in November 2002, and identified by Professor Kai-Jiao Jiang, Institute of Medicinal Plant Development, Chinese Academy of Medical Sciences \& Peking Union Medical College. A voucher specimen (EX97038) has been deposited at the Herbarium of Institute of Materia Medica, Chinese Academy of Medical Sciences \& Peking Union Medical College.

Extraction and Isolation The air-dried stem bark $(9.8 \mathrm{~kg})$ of Morus macaroura MiQ. was finely cut and extracted with refluxing EtOH successively (101, $3 \mathrm{~h}, 3$ times). Evaporation of the solvent under reduced pressure provided the ethanolic extract $(684.2 \mathrm{~g})$. The extract was dissolved and suspended in $\mathrm{H}_{2} \mathrm{O}(3.01)$, and partitioned with $\mathrm{CHCl}_{3}(3 \times 41)$, and EtOAc $(3 \times 41)$. The EtOAc fraction $(95.4 \mathrm{~g})$ was subjected to column chromatography on silica gel $(10 \times 120 \mathrm{~cm})$, eluted with $\mathrm{CHCl}_{3}$ and $\mathrm{MeOH}$ in increasing polarity, and were combined according to TLC monitoring to eight fractions. Fraction I $(1.8 \mathrm{~g})$ was chromatographed over reversed-phase silica gel [eluted by $\mathrm{MeOH}-\mathrm{H}_{2} \mathrm{O}, 30: 70-50: 50, \mathrm{v} / \mathrm{v}$ ] and purified by Sephadex $\mathrm{LH}-$ 20 [eluted by $\mathrm{MeOH}-\mathrm{H}_{2} \mathrm{O}, 50: 50, \mathrm{v} / \mathrm{v}$ ], to give guangsangon $\mathrm{K}(1,6 \mathrm{mg})$ and guangsangon $\mathrm{L}(\mathbf{2}, 5 \mathrm{mg})$. Fraction III $(3.3 \mathrm{~g})$ was subjected to reversedphase silica gel column chromatography [eluted by $\mathrm{MeOH}-\mathrm{H}_{2} \mathrm{O}, 40: 60-$ $50: 50, \mathrm{v} / \mathrm{v}]$ and purified by preparative TLC $\left(\mathrm{CHCl}_{3}-\mathrm{MeOH}, 7: 3, \mathrm{v} / \mathrm{v}\right)$ to give mulberrofuran $\mathrm{G}(\mathbf{3}, 8 \mathrm{mg})$, mulberrofuran $\mathrm{K}(4,15 \mathrm{mg})$ and a mixture ( $8 \mathrm{mg}$ ). The mixture was further separated by $\mathrm{C}_{18}$ HPLC (Alltech $\mathrm{C}-18$, $\left.250 \times 10 \mathrm{~mm}, \mathrm{MeOH}-\mathrm{H}_{2} \mathrm{O}, 35: 65, \mathrm{v} / \mathrm{v}\right)$ to give guangsangon $\mathrm{M}(\mathbf{5}, 4 \mathrm{mg}$, $\left.t_{\mathrm{R}}=26 \mathrm{~min}\right)$ and guangsangon $\mathrm{N}\left(6,2 \mathrm{mg}, t_{\mathrm{R}}=35 \mathrm{~min}\right)$.

Guangsangon $\mathrm{K}(\mathbf{1})$ : Brown amorphous powder, $[\alpha]_{\mathrm{D}}^{25}-178.5^{\circ}(c=0.14$, $\mathrm{MeOH})$. UV $\lambda_{\max }(\mathrm{MeOH}) \mathrm{nm}: 232(\mathrm{sh}), 278,319$ (sh). IR (KBr) $v_{\max } \mathrm{cm}^{-1}$. $3392,1678,1610,1508,1462$. FAB-MS $m / z: 627.4\left[\mathrm{M}+\mathrm{H}^{+}\right.$. HR-FAB-MS $m / z: 627.1873[\mathrm{M}+\mathrm{H}]^{+}$, (Calcd for $\mathrm{C}_{35} \mathrm{H}_{31} \mathrm{O}_{11}:$ 627.1866). $\mathrm{CD}$ $\left(c=7.19 \times 10^{-4} \mathrm{M}, \mathrm{MeOH}\right) \Delta \varepsilon(\mathrm{nm}): 282(-25.6), 287(-24.3), 347(+0.8)$. ${ }^{1} \mathrm{H}-\mathrm{NMR}\left(500 \mathrm{MHz}, \mathrm{CD}_{3} \mathrm{COCD}_{3}\right) \delta: 13.32\left(1 \mathrm{H}, \mathrm{s}, \mathrm{HO}-10^{\prime \prime}\right), 7.74(1 \mathrm{H}, \mathrm{d}$, $J=9.0 \mathrm{~Hz}, \mathrm{H}-5), 7.46\left(1 \mathrm{H}, \mathrm{d}, J=9.0 \mathrm{~Hz}, \mathrm{H}-14^{\prime \prime}\right), 7.09(1 \mathrm{H}, \mathrm{d}, J=8.5 \mathrm{~Hz}, \mathrm{H}-$ $\left.6^{\prime}\right), 6.88\left(1 \mathrm{H}, \mathrm{d}, J=9.0 \mathrm{~Hz}, \mathrm{H}-20^{\prime \prime}\right), 6.72(1 \mathrm{H}, \mathrm{d}, J=2.5 \mathrm{~Hz}, \mathrm{H}-8), 6.59(1 \mathrm{H}$ dd, $J=2.5,9.0 \mathrm{~Hz}, \mathrm{H}-6), 6.25\left(1 \mathrm{H}, \mathrm{d}, J=8.5 \mathrm{~Hz}, \mathrm{H}-5^{\prime}\right), 6.23(1 \mathrm{H}, \mathrm{d}$, $\left.J=2.5 \mathrm{~Hz}, \mathrm{H}-17^{\prime \prime}\right), 6.22\left(1 \mathrm{H}, \mathrm{dd}, J=2.0,9.0 \mathrm{~Hz}, \mathrm{H}-13^{\prime \prime}\right), 6.11(1 \mathrm{H}, \mathrm{dd}, J=2.5$, $\left.9.0 \mathrm{~Hz}, \mathrm{H}-19^{\prime \prime}\right), 5.96\left(1 \mathrm{H}, \mathrm{d}, J=2.0 \mathrm{~Hz}, \mathrm{H}-11^{\prime \prime}\right), 5.54\left(1 \mathrm{H}, \mathrm{s}, \mathrm{H}-2^{\prime \prime}\right), 5.48(1 \mathrm{H}$, d, $J=12.0 \mathrm{~Hz}, \mathrm{H}-2), 4.72(1 \mathrm{H}, \mathrm{d}, J=12.0 \mathrm{~Hz}, \mathrm{H}-3), 4.55\left(1 \mathrm{H}, \mathrm{br}, \mathrm{H}-4^{\prime \prime}\right)$, $4.53\left(1 \mathrm{H}\right.$, br s, H-3"), $3.72\left(1 \mathrm{H}\right.$, br s, H-5"), $2.67\left(1 \mathrm{H}, \mathrm{m}, \mathrm{H}-6^{\prime \prime}\right), 2.22(1 \mathrm{H}, \mathrm{m}$, $\left.\mathrm{H}-6^{\prime \prime}\right), 1.82\left(3 \mathrm{H}, \mathrm{s}, \mathrm{H}-7^{\prime \prime}\right) .{ }^{13} \mathrm{C}-\mathrm{NMR}$ data: see Table 1.

Guangsangon L (2): Yellow amorphous powder, $[\alpha]_{\mathrm{D}}^{25}-389.3^{\circ}(c=0.14$, $\mathrm{MeOH})$. UV $\lambda_{\max }(\mathrm{MeOH}) \mathrm{nm}: 236(\mathrm{sh}), 280$. IR (KBr) $v_{\max } \mathrm{cm}^{-1}: 3408$, $1674,1605,1512,1464$. FAB-MS $m / z: 477.2[\mathrm{M}+\mathrm{H}]^{+}$. HR-FAB-MS $m / z$ : $477.1558[\mathrm{M}+\mathrm{H}]^{+}$, (Calcd for $\left.\mathrm{C}_{27} \mathrm{H}_{25} \mathrm{O}_{8}: 477.1549\right) .{ }^{1} \mathrm{H}-\mathrm{NMR}(500 \mathrm{MHz}$, $\left.\mathrm{CD}_{3} \mathrm{COCD}_{3}\right) \delta: 13.04\left(1 \mathrm{H}, \mathrm{s}, \mathrm{OH}-10^{\prime \prime}\right), 11.19(1 \mathrm{H}, \mathrm{s}, \mathrm{OH}-4), 7.58(1 \mathrm{H}, \mathrm{d}$, $\left.J=9.0 \mathrm{~Hz}, \mathrm{H}-14^{\prime \prime}\right) 7.57(1 \mathrm{H}, \mathrm{s}, \mathrm{H}-6), 6.89\left(1 \mathrm{H}, \mathrm{d}, J=9.0 \mathrm{~Hz}, \mathrm{H}-20^{\prime \prime}\right), 6.20$ $\left(1 \mathrm{H}, \mathrm{d}, J=2.0 \mathrm{~Hz}, \mathrm{H}-17^{\prime \prime}\right), 6.12(1 \mathrm{H}, \mathrm{s}, \mathrm{H}-3), 6.10(1 \mathrm{H}, \mathrm{dd}, J=2.0,9.0 \mathrm{~Hz}, \mathrm{H}-$ $\left.19^{\prime \prime}\right), 6.09\left(1 \mathrm{H}, \mathrm{dd}, J=2.0,9.0 \mathrm{~Hz}, \mathrm{H}-13^{\prime \prime}\right), 5.97\left(1 \mathrm{H}, \mathrm{d}, J=2.0 \mathrm{~Hz}, \mathrm{H}-11^{\prime \prime}\right)$, $5.35\left(1 \mathrm{H}, \mathrm{s}, \mathrm{H}-2^{\prime \prime}\right), 4.42\left(1 \mathrm{H}, \mathrm{br} \mathrm{s}, \mathrm{H}-4^{\prime \prime}\right), 4.19\left(1 \mathrm{H}, \mathrm{br} \mathrm{s}, \mathrm{H}-3^{\prime \prime}\right), 3.63(1 \mathrm{H}, \mathrm{br} \mathrm{s}$, $\left.\mathrm{H}-5^{\prime \prime}\right), 2.23\left(1 \mathrm{H}, \mathrm{m}, \mathrm{H}-6^{\prime \prime}\right), 2.61\left(1 \mathrm{H}, \mathrm{m}, \mathrm{H}-6^{\prime \prime}\right), 1.77\left(1 \mathrm{H}, \mathrm{s}, \mathrm{H}-7^{\prime \prime}\right) .{ }^{13} \mathrm{C}-\mathrm{NMR}$ data: see Table 1 .

Guangsangon $\mathrm{M}(5)$ : Brown amorphous powder, $[\alpha]_{\mathrm{D}}^{25}-276.5^{\circ}(c=0.12$, $\mathrm{MeOH})$. UV $\lambda_{\max }(\mathrm{MeOH}) \mathrm{nm}: 230(\mathrm{sh}), 278,319(\mathrm{sh})$. IR (KBr) $v_{\max } \mathrm{cm}^{-1}$ : 3342, 1650, 1616, 1506, 1496, 1456, 1369. FAB-MS m/z: $611.2[\mathrm{M}+\mathrm{H}]^{+}$. HR-FAB-MS $m / z: 611.1962[\mathrm{M}+\mathrm{H}]^{+},\left(\right.$Calcd for $\mathrm{C}_{35} \mathrm{H}_{31} \mathrm{O}_{10}:$ 611.1917). $\mathrm{CD}$ $\left(c=8.20 \times 10^{-4} \mathrm{M}, \mathrm{MeOH}\right) \Delta \varepsilon(\mathrm{nm}): 285(-8.3), 305(+0.7), 326(-7.6)$. ${ }^{1} \mathrm{H}-\mathrm{NMR}\left(500 \mathrm{MHz}, \mathrm{CD}_{3} \mathrm{COCD}_{3}\right) \delta: 13.06\left(1 \mathrm{H}, \mathrm{s}, \mathrm{HO}-10^{\prime \prime}\right), 7.72(1 \mathrm{H}, \mathrm{d}$, $J=9.0 \mathrm{~Hz}, \mathrm{H}-5), 7.64\left(1 \mathrm{H}, \mathrm{d}, J=9.0 \mathrm{~Hz}, \mathrm{H}-14^{\prime \prime}\right), 7.39\left(1 \mathrm{H}, \mathrm{s}, \mathrm{H}-6^{\prime}\right), 6.85$ $\left(1 \mathrm{H}, \mathrm{d}, J=9.0 \mathrm{~Hz}, \mathrm{H}-20^{\prime \prime}\right), 6.68(1 \mathrm{H}, \mathrm{d}, J=2.5 \mathrm{~Hz}, \mathrm{H}-8), 6.52(1 \mathrm{H}, \mathrm{dd}, J=2.5$, $9.0 \mathrm{~Hz}, \mathrm{H}-6), 6.35\left(1 \mathrm{H}, \mathrm{dd}, J=2.0,9.0 \mathrm{~Hz}, \mathrm{H}-13^{\prime \prime}\right), 6.24\left(1 \mathrm{H}, \mathrm{s}, \mathrm{H}-3^{\prime}\right), 6.22$ $\left(1 \mathrm{H}, \mathrm{d}, J=2.5 \mathrm{~Hz}, \mathrm{H}-17^{\prime \prime}\right), 6.08\left(1 \mathrm{H}, \mathrm{dd}, J=2.5,9.0 \mathrm{~Hz}, \mathrm{H}-19^{\prime \prime}\right), 5.93(1 \mathrm{H}, \mathrm{d}$, $\left.J=2.0 \mathrm{~Hz}, \mathrm{H}-11^{\prime \prime}\right), 5.61(1 \mathrm{H}$, br d, $J=13.5 \mathrm{~Hz}, \mathrm{H}-2), 5.34\left(1 \mathrm{H}, \mathrm{s}, \mathrm{H}-2^{\prime \prime}\right), 4.53$ $(1 \mathrm{H}$, br s, H-4"), $4.36(1 \mathrm{H}$, br s, H-3"), $3.63(1 \mathrm{H}$, br s, H-5"), $2.96(1 \mathrm{H}, \mathrm{dd}$, $J=13.5,16.5 \mathrm{~Hz}, \mathrm{H}-3 \mathrm{a}), 2.72\left(1 \mathrm{H}, \mathrm{m}, \mathrm{H}-6^{\prime \prime}\right), 2.55(1 \mathrm{H}, \mathrm{br} \mathrm{d}, J=16.5 \mathrm{~Hz}, \mathrm{H}-$ 3b), $2.20\left(1 \mathrm{H}, \mathrm{m}, \mathrm{H}-6^{\prime \prime}\right), 1.73\left(3 \mathrm{H}, \mathrm{s}, \mathrm{H}-7^{\prime \prime}\right) .{ }^{13} \mathrm{C}-\mathrm{NMR}$ data: see Table 1

Guangsangon $\mathrm{N}(6)$ : Brown amorphous powder, $[\alpha]_{\mathrm{D}}^{21}-335.3^{\circ}(c=0.13$, $\mathrm{MeOH})$. UV $\lambda_{\max }(\mathrm{MeOH}) \mathrm{nm}: 206,231$ (sh), 276, $316(\mathrm{sh})$. IR (KBr) $v_{\max }$ $\mathrm{cm}^{-1}: 3406,1675,1616,1505,1456$. FAB-MS $m / z: 611.4[\mathrm{M}+\mathrm{H}]^{+}$. HRFAB-MS $m / z: 611.1958[\mathrm{M}+\mathrm{H}]^{+}$, (Calcd for $\mathrm{C}_{35} \mathrm{H}_{31} \mathrm{O}_{10}:$ 611.1917). $\mathrm{CD}$ $\left(c=8.20 \times 10^{-4} \mathrm{M}, \mathrm{MeOH}\right) \Delta \varepsilon(\mathrm{nm}): 269(-6.7), 288(-7.8), 303(-7.0)$, $333(+1.2) .{ }^{1} \mathrm{H}-\mathrm{NMR}\left(500 \mathrm{MHz}, \mathrm{CD}_{3} \mathrm{COCD}_{3}\right) \delta: 13.07\left(1 \mathrm{H}, \mathrm{s}, \mathrm{HO}-10^{\prime \prime}\right)$, $7.74(1 \mathrm{H}, \mathrm{d}, J=9.0 \mathrm{~Hz}, \mathrm{H}-5), 7.48\left(1 \mathrm{H}, \mathrm{d}, J=9.0 \mathrm{~Hz}, \mathrm{H}-14^{\prime \prime}\right), 7.38(1 \mathrm{H}, \mathrm{s}, \mathrm{H}-$ $\left.6^{\prime}\right), 6.87\left(1 \mathrm{H}, \mathrm{d}, J=9.0 \mathrm{~Hz}, \mathrm{H}-20^{\prime \prime}\right), 6.58(1 \mathrm{H}, \mathrm{dd}, J=2.5,9.0 \mathrm{~Hz}, \mathrm{H}-6), 6.54$ $(1 \mathrm{H}, \mathrm{d}, J=2.5 \mathrm{~Hz}, \mathrm{H}-8), 6.25\left(1 \mathrm{H}, \mathrm{d}, J=2.5 \mathrm{~Hz}, \mathrm{H}-17^{\prime \prime}\right), 6.20(1 \mathrm{H}, \mathrm{dd}, J=2.5$, $\left.9.0 \mathrm{~Hz}, \mathrm{H}-19^{\prime \prime}\right), 6.19\left(1 \mathrm{H}, \mathrm{s}, \mathrm{H}-3^{\prime}\right), 6.06\left(1 \mathrm{H}, \mathrm{dd}, J=2.0,9.0 \mathrm{~Hz}, \mathrm{H}-13^{\prime \prime}\right), 5.97$ $\left(1 \mathrm{H}, \mathrm{d}, J=2.0 \mathrm{~Hz}, \mathrm{H}-11^{\prime \prime}\right), 5.67(1 \mathrm{H}, \mathrm{brd}, J=13.0 \mathrm{~Hz}, \mathrm{H}-2), 5.35(1 \mathrm{H}, \mathrm{s}, \mathrm{H}-$ $\left.2^{\prime \prime}\right), 4.31(1 \mathrm{H}$, br s, H-4"), $4.23(1 \mathrm{H}$, br s, H-3"), 3.61 (1H, br s, H-5"), 3.04 $(1 \mathrm{H}, \mathrm{dd}, J=13.9,18.0 \mathrm{~Hz}, \mathrm{H}-3 \mathrm{a}), 2.73\left(1 \mathrm{H}, \mathrm{m}, \mathrm{H}-6^{\prime \prime}\right), 2.61(1 \mathrm{H}$, brd, $J=18.0 \mathrm{~Hz}, \mathrm{H}-3 \mathrm{~b}), 2.19\left(1 \mathrm{H}, \mathrm{m}, \mathrm{H}-6^{\prime \prime}\right), 1.76\left(3 \mathrm{H}, \mathrm{s}, \mathrm{H}-7^{\prime \prime}\right) .{ }^{13} \mathrm{C}-\mathrm{NMR}$ data: see Table 1 .

Anti-oxidation Bioassays The anti-oxidant activities of 1, 2, 5, 6 and Vit $\mathrm{E}$ were determined by the content of MDA (malondialhyde), a compound that was produced during microsomal lipid per-oxidation induced by ferrous-cysteine. MDA was detected by using the thiobarbituric acid (TBA) method. Briefly, $1 \mathrm{mg} / \mathrm{ml}$ microsomal protein, different concentration compound or vehicle and $0.2 \mathrm{~mm}$ cysteine in $0.1 \mathrm{~m}$ PBS were incubated for $15 \mathrm{~min}$ at $37^{\circ} \mathrm{C}$, added $0.5 \mathrm{~mm}$ ferrous, mixed and incubated for $15 \mathrm{~min}$ at $37^{\circ} \mathrm{C}$ again. The equal volume of $20 \%$ TCA was added to terminate the reaction and centrifuged for $10 \mathrm{~min}$ at $3000 \mathrm{rpm}$. The supernatants reacted with $0.67 \% \mathrm{TBA}$ for $10 \mathrm{~min}$ at $100^{\circ} \mathrm{C}$. After cooling, the MDA was quantified by determining the absorbance at $532 \mathrm{~nm}$, and then calculated the inhibition rate.

\section{References}

1) Sun S.-G., Chen R.-Y., Yu D.-Q., J. Asian Nat. Prod. Res., 3, 253-259 (2001).

2) Nomura T., Fukai T., Junko M., Imashimizu A., Terada S., Hama M., Planta Medica, 46, 167-174 (1982).

3) Nomura T., Fukai T., Hano T., Nemoto K., Terada S., Kuramochi T., Planta Medica, 47, 151-156 (1983).

4) Hano Y., Hirakura K., Nomura T., Terada S., Fukushima K., Planta Medica, 48, 127-130 (1984).

5) Hirakura K., Fukai T., Hano Y., Nomura T., Phytochemistry, 24, 159161 (1985).

6) Hano Y., Aida M., Nomura T., J. Nat. Prod., 53, 391-395 (1990).

7) Shen R.-C., Lin M., Phytochemistry, 57, 1231-1235 (2001). 
8) Hiracura K., Hano Y., Fukai T., Nomura T., Uzawa J., Fukushima K., Chem. Pharm. Bull., 33, 1088-1096 (1985).

9) Sherif C. A., Gupta R. K., Krishnamurti M., Tetrahedron Lett., 21, 641-642 (1980).

10) Hano Y., Suzuki S., Nomura T., Iitaka Y., Heterocycles, 27, 23152324 (1988).

11) Gaffield W., Tetrahedron, 26, 4093-4108 (1970).
12) Hano Y., Suzuki S., Kohno H., Nomura T., Heterocycles, 27, 75-81 (1986).

13) Snatzke G., Znatke F., Tetrahedron, 29, 909-912 (1973).

14) Fukai T., Hano Y., Hirakura K., Nomura T., Fukushima K., Chem. Pharm. Bull., 33, 3195-3204 (1985).

15) Hano Y., Kohno H., Nomura T., Chem. Pharm. Bull., 33, 5294-5300 (1985). 